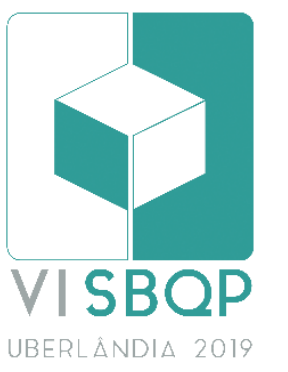

\title{
UM MAPEAMENTO SISTEMÁTICO DA LITERATURA: STAKEHOLDERS NO UPGRADING DE HABITAÇÕES
}

\author{
DALPINO, Lucas Basaglia \\ Universidade Estadual de Campinas, e-mail: lucasdalpino@gmail.com \\ KOWALTOWSKI, Doris Catharine Cornelie Knatz \\ Universidade Estadual de Campinas, e-mail: doris@fec.unicamp.br \\ GRANJA, Ariovaldo Denis \\ Universidade Estadual de Campinas, e-mail: adgranja@fec.unicamp.br
}

\begin{abstract}
RESUMO
Este artigo, produto de disciplina, busca estruturar a produção acadêmica de maior expressão por meio do método de Mapeamento Sistemático da Literatura (MSL). O uso do MSL aliado às ferramentas facilitadoras para seleção de publicações aderentes ao tema central de pesquisa, tem como objetivo obter evidências a partir de estudos primários já publicados e identificar eventuais lacunas relacionadas às atuações das partes interessadas na construção civil, focada no upgrading das habitações. Nesta pesquisa, partes interessadas (stakeholders) são definidos como qualquer grupo ou indivíduo que afetam ou são afetados pela realização de objetivos de um empreendimento. A temática merece atenção da comunidade científica, uma vez que se pressupõe nesta pesquisa que possa haver alinhamento entre os resultados almejados por empresas provedoras de habitações e o acréscimo de entrega de valor aos seus usuários. Os resultados do MSL indicam uma maioria de estudos relacionada aos processos de negócios e de tomada de decisão, com evidências de crescimento de produção nos últimos sete anos. Verifica-se a contribuição do MSL ao eleger 17 artigos correlatos ao foco temático a partir de um cenário preliminar com 268 textos. Esta redução da quantidade de artigos define qualitativamente os resultados dos objetos de pesquisa encontrados, favorecendo a produção acadêmica.
\end{abstract}

Palavras-chave: Mapeamento sistemático; Partes interessadas; Edifícios residenciais; Renovação; Revitalização.

\begin{abstract}
This article, the product of discipline, seeks to structure the academic production of greater expression through the method of Systematic Mapping of Literature (MSL). The use of the MSL, together with the facilitating tools to select publications adhering to the central research theme, aims to obtain evidence from previously published primary studies and identify any gaps related to the actions of the stakeholders in the construction industry, focused on the upgrading of housing. In this research, stakeholders are defined as any group or individual that affects or is affected by the achievement of the objectives of an enterprise. The theme deserves attention from the scientific community, since it presupposes in this research that there may be an alignment between the results sought by housing companies and the increase of value delivery to their users. The results of MSL indicate a majority of studies related to business processes and decision making, with evidence of production growth in the last seven years. The contribution of the MSL in selecting 17 articles correlated to the thematic focus is verified from a preliminary scenario with 268 texts. This reduction in the number of articles qualitatively defines the results of the research objects found, favoring academic production.
\end{abstract}

Keywords: Systematic mapping study; Stakeholder; Residential buildings; Retrofit; Upgrading.

DALPINO, L. B.; KOWALTOWSKI, D. C. C. K.; GRANJA, A. D. Um Mapeamento Sistemático da Literatura: Stakeholders no Upgrading de Habitações. In: SIMPÓSIO BRASILEIRO DE QUALIDADE DO PROJETO NO AMBIENTE CONSTRUÍDO, 6., 2019, Uberlândia. Anais... Uberlândia: PPGAU/FAUeD/UFU, 2019. p. 239-247. DOI https://doi.org/10.14393/sbqp19024. 


\section{INTRODUÇÃO}

Durante muito tempo, assumiu-se que os pontos de vista dos stakeholders, as partes interessadas (PI) no negócio, poderiam ser tratados como externalidades para o planejamento estratégico (Freeman, 1983). Passados quase quarenta anos, o pensamento administrativo tem mudado drasticamente, como reconhecido por Freeman em 1983. De forma conceitual e prática pode ser considerado no setor da construção civil devido a um mercado atual instável, com altos índices de vacância nas edificações residenciais verificados pelo SECOVI-SPI que indicava 22.327 unidades residenciais em estoque no ano de 2018 ante as 22.040 levantadas em 2017, além das crescentes inadimplências nos pagamentos dos imóveis, que apontavam 22\%, em junho de 2015, considerando apenas a Faixa 1 do Programa Minha Casa, Minha Vida² (Fraga; Vieira, 2016).

O ponto de partida de qualquer análise das PI é a identificação dos envolvidos, sem que exista determinação única (Freeman, 1983). As Pl podem ser composta por fornecedores, parceiros, aliados, clientes (Freeman; Liedtka, 1997), entre outros, desde que ligados aos temas centrais da estratégia de negócio (Freeman; Liedtka, 1997). O stakeholder é qualquer grupo ou indivíduo que possa afetam ou são afetados pela realização dos objetivos da organização, definição tradicional segundo Freeman (1984).

Embora existam programas que orientem alguns alinhamentos, os envolvidos estão sujeitos às disfunções ou ineficiências (Freeman 1984, p.150), dessa forma ainda há muito trabalho a ser feito pelos executivos e pensadores para aguçarem e moldarem os focos em novas visões de negócios e novos modelos estratégicos (Freeman; Liedtka, 1997) que considerem a maioria das Pl envolvidas possíveis; intuito da atual pesquisa baseada em evidências.

Buscando evidências de melhor qualidade em estudos científicos a respeito de tópico específico (Kitchenham et al. 2008), como o caso da atuação dos stakeholders na construção civil, é proposto nesse artigo um Mapeamento Sistemático da Literatura (MSL) para relacionar as pesquisas e resultados que já foram publicados, categorizando-os (Petersen et al. 2008). Visto que os MSL estão principalmente preocupados em agregar evidências empíricas de diferentes origens e contextos amplamente diferentes (Brereton et al. 2006), é importante mencionar que um MSL também ajuda a identificar lacunas de pesquisa em uma área temática (Petersen et al. 2008).

\section{MÉTODO DE PESQUISA}

O MSL é geralmente usado como o primeiro passo em direção a uma revisão sistemática, ou seja, primeiro a área temática é estruturada e, posteriormente, uma área específica é investigada com uma revisão sistemática (Petersen et al. 2008).

Assim sendo, o MSL pode ser utilizado com o objetivo de identificar a extensão e a forma da literatura sobre um tópico em particular (Bailey et al., 2007), antecedendo a Revisão Sistemática da Literatura (RSL). A RSL é uma forma de

\footnotetext{
${ }^{1}$ Sindicato das Empresas de Compra, Venda, Locação e Administração de Imóveis Residenciais e Comerciais de São Paulo.

2 O Minha Casa, Minha Vida (MCMV) foi um programa federal brasileiro lançado em 2009, pelo Governo Lula, que subsidia a compra de imóveis residenciais para famílias com renda de até 1,8 mil reais e facilita as condições de aquisição para outras com rendimentos máximos de 9 mil. Existem cinco modalidades, sendo a Faixa 1 destinadas às famílias com as menores rendas - até 1,8 mil reais.
} 
identificar, avaliar e interpretar toda a pesquisa relevante disponível em determinadas bases para uma questão de pesquisa específica, área temática ou fenômeno de interesse (Kitchenham, 2004), sendo essa uma etapa considerada posterior ao MSL, quando é pretendido aprofundamento nos resultados prévios obtidos.

O intuito das revisões é comparar relatórios primários existentes, gerados pela SMS, analisá-los em profundidade e descrever sua metodologia e resultados (Petersen et al. 2008), beneficiando a definição da metodologia (Kitchenham; Charters, 2007). Tende a ajudar na uniformidade de codificação (Brereton et al. 2006) e considera uma gama mais ampla de situações e contextos (Kitchenham; Charters, 2007), focando em resultados mais embasados e assertivos. Dessa forma, esse artigo, propõe seguir a aplicação de tais processos de acordo com o fluxograma representado pela Figura 1, inspirado em Kitchenham (2004):

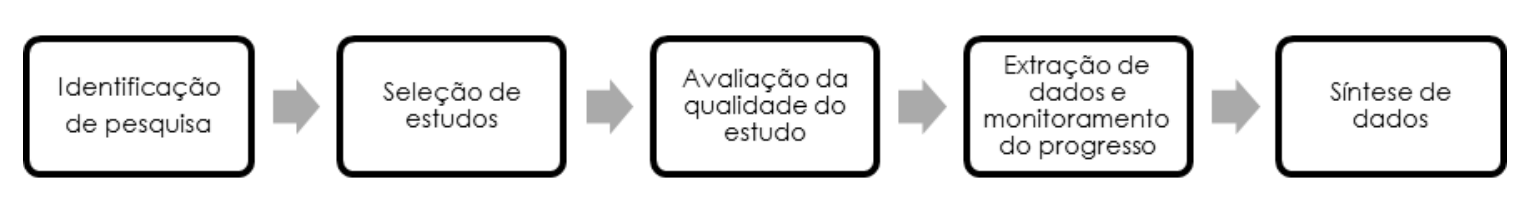

Figura 1 - Fluxograma para condução do MSL

Fonte: Autores (2019)

\subsection{Identificação da pesquisa}

Especificar as questões de pesquisa é a parte mais importante de qualquer revisão sistemática (Kitchenham; Charters, 2007), já que tendem a conduzir toda a metodologia empregada e definir o tema de investigação. Lembrando ser necessária a determinação de uma estratégia de busca (Kitchenham, 2004).

Dessa forma, a questão de pesquisa que norteou este MSL é: "quais os papéis estratégicos dos stakeholders no upgrading de habitações?", selecionando as palavras "stakeholders" e sinônimos da palavra requalificação como "retrofit", "renovação" e "revitalização", e a fim de direcionar os resultados das buscas aos edifícios habitacionais, também houve a opção pela sua adoção. Sendo essa combinação de termos responsáveis por buscar resultados relevantes de estudos anteriores que abordam o tema central da pesquisa e 0 detalhamento almejado, sem que fosse alterada depois de sua definição (Brereton et al. 2006).

\subsection{Seleção de estudos}

A estratégia usada para pesquisar estudos primários pode considerar, além dos termos de pesquisa, as ferramentas que localizam os recursos a serem desenvolvidos e incluem bibliotecas digitais, periódicos específicos e anais de conferências (Kitchenham; Charters, 2007), passíveis de buscas através das strings em Bases de Dados.

Levando-se em consideração esta abordagem foram escolhidas três Bases de Dados relacionadas com a área de estudo para as pesquisas do SMS: Scopus, Web of Knowledge e Engineering Village, consideradas bases de qualidade reconhecida para os propósitos deste MSL. A string de busca considera resultados de palavras localizados no resumo e no título, ou ainda em outras partes, além de opção por outros filtros como restrições de datas. 


\section{AVALIAÇÃO DA QUALIDADE DO ESTUDO}

Para determinação da string de pesquisa utilizada, foram selecionadas palavras-chaves em publicações relacionadas ao tema no Google Acadêmico e, também, indicadas nas referências das mesmas, já que o MSL inicial pode ajudar a determinar estratégias apropriadas (Kitchenham; Charters, 2007). Dessa forma conduziram-se algumas pesquisas preliminares destinadas a identificar as revisões sistemáticas existentes e avaliar o volume de estudos potencialmente relevantes, além de pesquisas de teste usando várias combinações de termos derivados da questão de pesquisa, conforme sugerido por Kitchenham e Charters (2007). Foram utilizados, além de sinônimos ou expressões semelhantes (Brereton et al. 2006), os operadores booleanos OR e AND (Kitchenham, 2004) - e truncamentos (*) com intuito em ampliar a gama de resultados a serem obtidos, desconsiderando as terminologias das palavras. Verificou-se, no entanto, a expressiva quantidade de palavras com significado de "requalificação", assim foram elencadas, também, "retrofit", "renovação" e "revitalização".

Além da consideração das especificidades de pesquisa em cada base de dados adotada e das variantes em torno das palavras-chaves que melhor exprimiram e representariam o tema de pesquisa, foi necessário encontrar termos em língua inglesa que o traduzisse, já que é o idioma difundido globalmente em artigos científicos, portanto, depois de algumas tentativas, a melhor determinação encontrada, que respeitasse os recursos disponibilizados pelas bases e suas especificidades, para a string de busca foi: "stakeholders AND hous* OR building AND retrofit OR renovation OR revitalization".

Houve, como forma de restringir os dados de saída e obter maior confiabilidade nos resultados, com possibilidade de adoção de critérios de inclusão e exclusão, admitindo apenas temas relacionados ao mercado da construção civil e seus negócios e, também, exclusivamente artigos independentes do ano de publicação.

\subsection{Extração de dados e monitoramento do progresso}

Todos os resultados obtidos nas pesquisas, incluindo seus critérios iniciais de seleção, foram salvos de acordo com os métodos disponibilizados pelas Bases de Dados utilizadas. Desse modo os recursos disponíveis para se salvar os arquivos e as informações foram, também, determinantes para suas definições.

Diante de um cenário de possibilidades de transferências, encontrou-se a transferência individual do arquivo, comumente feita com extensão "pdf", quando há disponibilidade do arquivo; arquivamento dentro da Base de Dados, quando há registro específico de usuário; envio dos resultados por email, em formato "html" ou formato "txt"; ou uso de softwares específicos que administram as referências bibliográficas como o "Zotero" e o "Endnote", segundo levantado por Ruiz et al. (2013).

Sendo possível analisar a quantidade de extensões distintas de saídas de cada uma das Bases, variando de extensões genéricas de fácil acesso e leitura até outras mais específicas e reconhecidas por softwares destinados a tal uso, e se mesmo assim não satisfizerem os anseios do pesquisador é ainda possível selecionar os dados pertinentes manualmente, com seleção das informações dos resultados da pesquisa e cópia em arquivo de planilha eletrônica, como orientado por Petersen et al. (2008), de preferência do usuário e que facilite a manipulação, ordenação e sistematização das informações obtidas em cada 
registro.

Verificou-se também a possibilidade de registros diretos em nuvens de armazenamento dados, além dos tradicionais computadores pessoais.

Parametrizando essas informações e preferências pessoais, os dados obtidos na pesquisa em curso foram salvos em extensão específica de "BibTeX", considerando informações completas do artigo, como título, autor, ano, fonte de publicação e seu resumo, além do próprio documento quando disponível em "pdf" para importação através do sistema operacional "zotero", que com abertura de conta pessoal possibilitou o armazenamento dos arquivos em nuvens com acessos regulares em diferentes localidades e equipamentos.

Dessa forma verificou-se que o processo de extração de dados visa extrair os itens necessários para responder questões (Kitchenham; Charters, 2007) envolvendo o tema de pesquisa em eventual análise posterior.

\subsection{Síntese de dados}

Uma das formas primárias de análise indicada é a supressão dos arquivos duplicados, já que por terem sido pesquisados em três Bases de Dados diferentes, nesse caso, podem ter encontrado resultados idênticos, uma vez que houve padronização da string de busca entre elas - salvo especificidades de cada base. Podendo ser feita manualmente ou explorando a opção semiautomática oferecida por alguns sistemas operacionais de gerenciamento de referências bibliográficas, como é o caso do "Zotero", adotado nessa sistematização.

O segundo passo possível é a exclusão através das análises dos títulos dos artigos encontrados e listados ainda no gerenciador, quando muito destoante do que se busca.

Dessa forma, a quantidade de resultados iniciais deve apresentar considerável redução, que possibilitará uma organização que exige maior demanda de atenção, foco e trabalho mais minucioso nas análises. Os arquivos resultantes da pesquisa foram organizados em uma planilha eletrônica, já que tabular os dados é considerado um meio útil de agregação e necessários para responder às questões de pesquisa (Brereton et al. 2006), com destaque para a origem da base de cada arquivo e as datas de levantamento dos dados. Cabe ressaltar que os estudos de MSL podem ser conduzidos com base apenas nos resumos, no entanto, Petersen et al. notou que os resumos costumam ser enganosos e carecem de informações importantes (2008).

Como é necessário maior grau de detalhamento, criaram-se colunas em planilhas eletrônicas como: "Aderente", quando o artigo está de acordo com a referência desejada; "Identificação", codificando cada arquivo de acordo seu número dentro da Base de Dados; "Palavras-Chave", citando-as; "Títulos, nomes completos extraídos de cada texto de acordo com os dados de saída; "Resumos", compondo maior detalhamento dos resultados através de leituras; "Ano de Publicação"; Meio de Publicação"; "Tipo de Estudos" (Kitchenham, 2004); como adotadas nesse mapeamento ou outras conforme a necessidade e desejo de cada pesquisador para que o processo de análise possa sintetizar os dados de tal forma que as perguntas possam ser respondidas (Kitchenham; Charters, 2007) e aprofundadas com as leituras completas dos artigos correlatos. 


\section{RESULTADOS}

A pesquisa inicial resultou em 894 artigos preliminares, que esclarecem 0 cenário de publicações existentes relacionadas aos "papéis estratégicos dos stakeholders no upgrading de habitações", que após seleção relacionada ao tema de interesse, houve uma redução de 423 arquivos, que submetidos a uma nova filtragem, agora exclusivas aos artigos, obtive-se 268 (Figura 2). Como descrito por Petersen et al. (2008), que durante o MSL uma quantidade maior de artigos pode ser analisada, já que não precisam ser avaliados com detalhes.

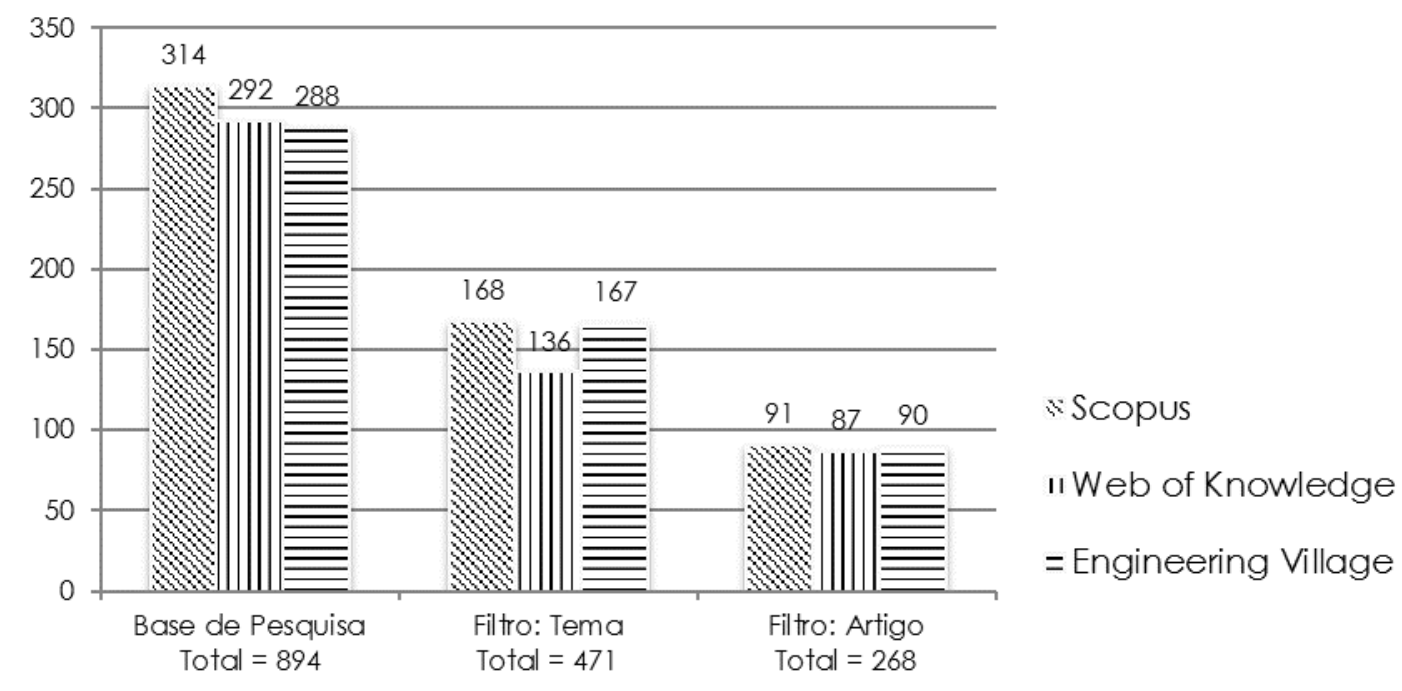

Figura 2 - Distribuição dos Textos nas Bases de Dados Fonte: Autores (2019)

As publicações selecionadas nas bases de dados (Scopus, Web of Knowledge e Enginnering Village) foram exportadas no formato "ris" para o software Zotero, classificados em pastas específicas. Na sequência, eliminou-se os artigos duplicados, o que gerou uma redução de 268 para 155 publicações ver Quadro 2. Esse resultado foi considerado passível de terem seus aspectos bibliométricos analisados em etapas seguintes.

Com um cenário quase que 17\% menor que o inicial (de 894 para 155), os 155 arquivos foram analisados conforme aderência, de acordo com os títulos, e reduzidos a um total de 26 artigos (Quadro 2). O fato de os artigos não serem minuciosamente avaliados podem gerar erros de julgamento ao classificar os artigos em categorias detalhadas e podem ser minimizadas em revisões sistemáticas posteriores (Petersen et al. 2008).

Ainda foram aplicados critérios de inclusão e de exclusão (Quadro 1), que reduziram para 17 artigos pertinentes ao tema de pesquisa, de um total de 155 antes dos refinamentos, ou seja, 89\%. É importante avaliar a qualidade dos estudos primários e seus critérios de inclusão e exclusão, ponderações a estudos específicos durante o estágio de síntese (Brereton et al. 2006), além do uso da tecnologia ressalta-se o refino particular de cada revisor. 


\section{Quadro 1 - Critérios de inclusão e exclusão}

\begin{tabular}{|c|c|}
\hline Inclusão & Exclusão \\
\hline $\begin{array}{c}\text { Edifícios habitacionais; sustentabilidade em retrofit; } \\
\text { riscos em negócios da construção; impactos sociais; } \\
\text { custos efetivos; impactos e políticas nas } \\
\text { oportunidades de retrofit; restauro urbanos; } \\
\text { renovação de edifícios. }\end{array}$ & $\begin{array}{c}\text { Edifícios comerciais, industriais e hospitalares; } \\
\text { veículos; eficiência energética, térmica ou } \\
\text { hidráulica; BIM; sustentabilidade específica e } \\
\text { LEED; abalos sísmicos e inundações; sociologia, } \\
\text { pedagogia e odontologia; reformas individuais. }\end{array}$ \\
\hline
\end{tabular}

Fonte: Autores (2019)

Os dados dos artigos foram novamente exportados, e complementados manualmente, quando necessários, para uma planilha eletrônica. Assim, procedeu-se a etapa de leitura e análise dos resumos, o que resultou na eliminação de nove publicações (Quadro 2). As eliminações confirmaram que as etapas básicas da sistemática de revisão são bastante relevantes (Brereton et al. 2006). Essa classificação ajuda na economia de tempo em análises posteriores (Kitchenham; Charters, 2007).

\section{Quadro 2 - Redução do resultado total de textos aos aderentes}

\begin{tabular}{|c|c|c|c|}
\hline $\begin{array}{c}\text { Resultado total de } \\
\text { textos }\end{array}$ & $\begin{array}{c}\text { Textos sem } \\
\text { duplicidade }\end{array}$ & $\begin{array}{c}\text { Textos depois da } \\
\text { análise dos títulos }\end{array}$ & $\begin{array}{c}\text { Textos depois da } \\
\text { leitura dos resumos }\end{array}$ \\
\hline 268 & 155 & 26 & 17 \\
\hline
\end{tabular}

Fonte: Autores (2019)

As análises específicas dos artigos relacionados permitem agrupá-los e classificá-los, a partir da leitura de seus títulos e resumos, sendo possível verificar que o tema consegue ser apresentado de diferentes métodos de pesquisa, desde os mais de 70\% (12 de 17) apresentados como estudo de caso até manifestações únicas como em experimentos (Figura 3).

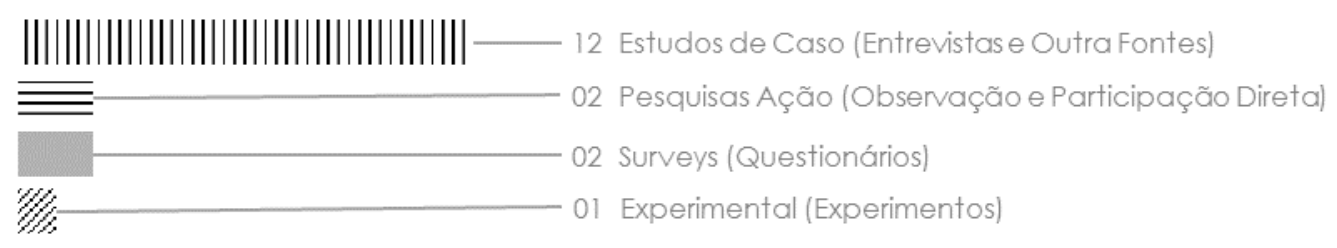

Figura 3 - Classificação dos Textos selecionados conforme Métodos

Fonte: Autores (2019)
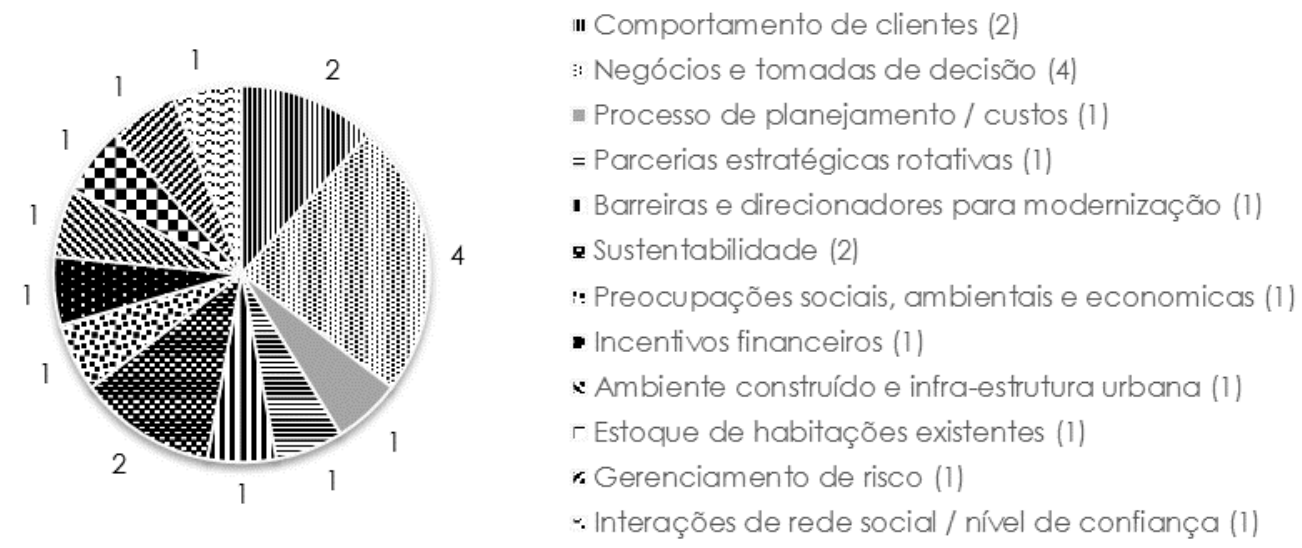

II Comportamento de clientes (2)

:: Negócios e tomadas de decisão (4)

- Processo de planejamento / custos (1)

= Parcerias estratégicas rotativas (1)

- Barreiras e direcionadores para modernização (1)

- Sustentabilidade (2)

: Preocupações sociais, ambientais e economicas (1)

- Incentivos financeiros (1)

- Ambiente construído e infra-estrutura urbana (1)

$\ulcorner$ Estoque de habitações existentes (1)

* Gerenciamento de risco (1)

- Interações de rede social / nível de confiança (1)

Figura 4 - Distribuição dos campos de conhecimento dos textos selecionados Fonte: Autores (2019) 
Através das classificações dos resultados encontrados foi possível notar a multidisciplinaridade abordada nos artigos, sendo que do montante final inerente aos estudos desejados foi identificado que $24 \%$ da produção, ou seja, 4/17 está enquadrado no campo de conhecimento de "negócios e tomadas de decisão" (Figura 4), sendo esse ponto incomum para o avanço de pesquisas direcionadas.

om os MSL os artigos não avaliados quanto à sua qualidade (Petersen et al. 2008), em Base de Dados e o processo de aplicação do SMS foi possível perceber a recente produção e abordagens (Figura 4) que aborda os "os papéis estratégicos dos stakeholders no upgrading de habitações", sendo a primeira publicação acerca do assunto datada de 2009, e todas as demais concentradas nos últimos sete anos, em 11 diferentes periódicos (Figura 5).

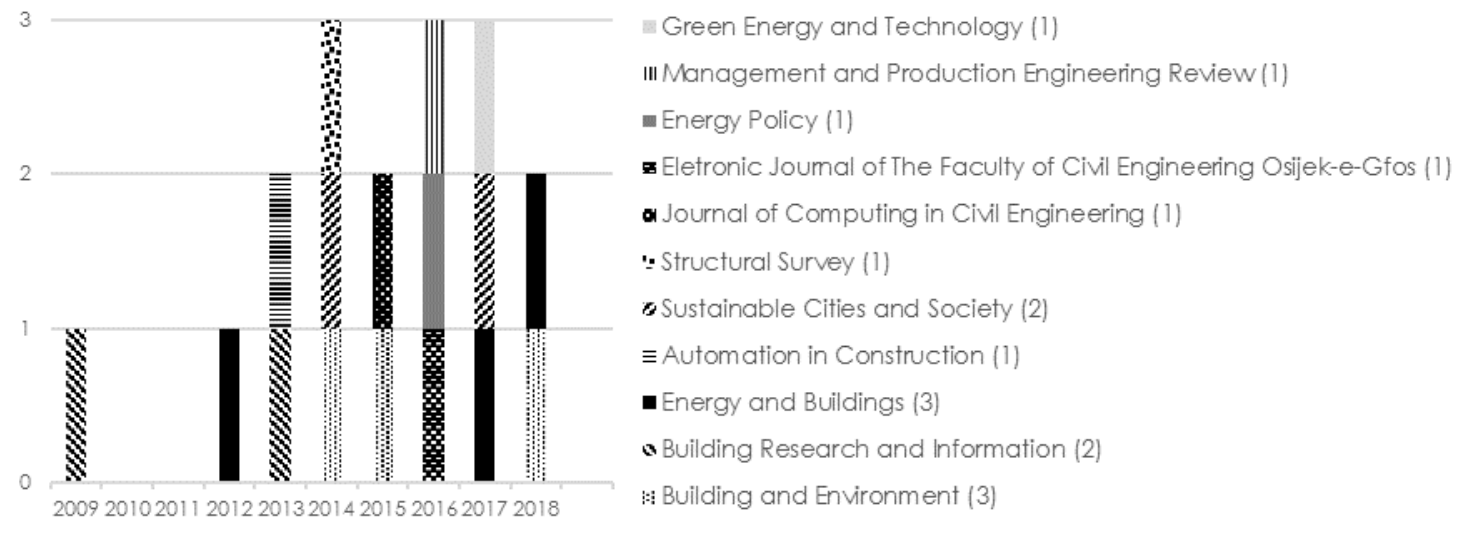

Figura 5 - Distribuição dos Textos selecionados por ano e seus Periódicos Fonte: Autores (2019)

\section{CONCLUSÕES}

Este artigo retratou os esforços iniciais de um MSL sobre o que já foi publicado em relação aos "papéis estratégicos dos stakeholders no upgrading de habitações", referente à problematização e à questão de pesquisa deste estudo.

As buscas, adotando-se o MSL proposto, possibilitaram alinhamento e acesso às produções científicas, num total de 26 publicações aderentes, como forma de ampliar os embasamentos bibliográficos e auxiliar na garantia de trabalhos inovadores.

É ainda observada a possibilidade de existirem limitações nos resultados devido aos filtros disciplinares aplicados, quantidade restrita de Bases de Dados consultadas, ou palavras-chave posicionadas além do título ou resumo.

Dessa forma, esse artigo elencou no processo final de filtragem 17 textos cerca de $2 \%$ do total inicial (Figura 2 e Quadro 2) - que se aproximam do objeto de pesquisa, sem delimitação de datas de publicação, sendo todos artigos de 11 períodos distintos (Figura 5), classificados em 12 campos de conhecimento (Figura 4), sem o uso da "amostragem bola de neve3" que tende a aprofundar ainda mais as pesquisas e aumentar a qualidade dos artigos referentes ao tema central apurado para investigações posteriores.

Este artigo presume que pesquisadores de áreas distintas possam se valer do

${ }^{3}$ A amostragem de bola de neve é uma metodologia não probabilística, que utiliza cadeias de referência, discutida por Handcock et al. (2011) e comumente usada para populações de difícil acesso, podendo ser benéfica em problemas de pesquisa específicos, considerando-se suas limitações. 
MSL para aprimorarem suas referências bibliográficas com certa qualidade, organização e rigor metodológico.

\section{REFERÊNCIAS}

BAILEY, J.; BUDGEN, D.; TURNER, M.; KITCHENHAM, B.; Brereton, P.; LINKMAN, S. Evidence relating to object-oriented software design: A survey, in 'Proc. of the 1st Int. Symp. on Empirical Software Engineering and Measurement (ESEM 2007), pp. 482-484. 2007.

BRERETON, Pearl; KITCHENHAM, A. Barbara; BUDGEN, David; TURNER, Mark; KHALILC, Mohamed. Lessons from applying the systematic literature review process within the software engineering domain. 2006.

ÉPOCA NEGÓCIOS. Venda de imóveis cresce $\mathbf{2 7 \%}$ na cidade de São Paulo em 2018, diz Secovi-SP. Publicado em 20/02/2019. Disponível em:

$<$ https://epocanegocios.globo.com/Mercado/noticia/2019/02/epocanegocios-venda-de-imoveis-cresce-27-na-cidade-de-sao-paulo-em-2018-dizsecovi-sp.html>. Acessado em 20 de fevereiro de 2019.

FRAGA, Luana dos Santos; VIEIRA, Kelmara Mendes. Quem são os inadimplentes do Programa Minha Casa Minha Vida?: Analisando a associação da inadimplência com variáveis socioeconômicas e de gerenciamento financeiro. FGV EAESP - GVcef - $03^{\circ}$ Encontro Brasileiro de Economia e Finanças Comportamentais. 2016.

FREEMAN, R. E.; REED, D. L. Stockholders and stakeholders: a new perspective on corporate governance. California Management Review, California: ABI/Inform, v. 25, n. 3, p. 88-92, Spring 1983.

FREEMAN, E. R. Strategic management- a stakeholder approach. London: Pitman Publishing. 1984.

FREEMAN, R. E.; LIEDTKA, J. Stakeholder capitalism and the value chain. European Management Journal, Amsterdam: Elsevier, v. 15, n. 3, p. 286-296, June 1997.

HANDCOCK, Mark S.; GILE, Krista J. Comment: on the Concept of Snowball Sampling. 2011.

KITCHENHAM, Barbara. Procedures for Performing Systematic Reviews. 2004.

KITCHENHAM, B; CHARTERS, Stuart. Guidelines for performing Systematic Literature Reviews in software engineering. EBSE Technical Report EBSE. 2007.

KITCHENHAM, Barbara; BRERETON, O. Pearl; BUDGEN, David; TURNER, Mark; BAILEY, John; LINKMAN, Stephen Linkman. Systematic literature reviews in software engineering - A systematic literature review. 2008.

PETERSEN, Kai; FELDT, Robert; MUJTABA, Shahid; MATTSSON, Michael. Systematic Mapping Studies in Software Engineering. 2008.

RUIZ, Joyce de Andrade; GRANJA, Ariovaldo Denis. Um mapeamento sistemático da literatura sobre a relação entre valor e colaboração na construção. 2013. 